\title{
Expression of duck hepatitis A virus type 1 VP3 protein mediated by avian adeno-associated virus and its immunogenicity in ducklings
}

\author{
A. P. WANG, L. LIU, L. L. GU, S. WU, C. M. GUO, Q. FENG, W. L. XIA, C. YUAN, S. Y. ZHU*
}

Jiangsu Agri-animal Husbandry Vocational College, Jiangsu Key Laboratory for High-Tech Research and Development of Veterinary Biopharmaceuticals, 8 East Phoenix Road, Taizhou, Jiangsu Province, 225300, P. R. China

Received May 21, 2018; revised June 29, 2018; accepted January 5, 2019

\begin{abstract}
Summary. - The avian adeno-associated virus (AAAV) is a replication-defective nonpathogenic virus that has been proved to be useful as a viral vector in gene delivery. In this study, the feasibility of AAAV for transgenic expression of duck hepatitis A virus (DHAV) VP3 structural protein and its ability to induce protective immunity in ducklings was assessed. The recombinant AAAV (rAAAV-VP3) expressing the VP3 protein was prepared by co-infection of Sf9 cells with recombinant baculovirus (rBac-VP3) containing VP3 gene flanked by inverted terminal repeats (ITRs) of AAAV and the other two recombinant baculovirus expressing AAAV functional and structural genes, respectively. The generation of rAAAV-VP3 was demonstrated by electron microscopy, immunofluorescence assay, and western blot analysis. One day old ducklings were inoculated with rAAAV-VP3 or commercial attenuated vaccine and then challenged with DHAV-1 strain SH two weeks post vaccination. Anti-DHAV-1 antibodies were detected in all vaccinated groups by ELISA, and the titers between the rAAAV-VP3 group and the attenuated vaccine group were not statistically significant. Real time RT-PCR analysis showed that the virus copy numbers in the livers of the PBS control group were significantly higher than that of the rAAAV-VP3 and attenuated vaccine groups. In conclusion, we demonstrated that the VP3 expression mediated by rAAAV in ducklings could induce protective immunity against DHAV challenge, and this could be a candidate vaccine for the control of duck viral hepatitis.
\end{abstract}

Keywords: avian adeno-associated virus; duck hepatitis A virus; VP3 gene; immunogenicity

\section{Introduction}

Duck viral hepatitis (DVH), caused by duck hepatitis A virus (DHAV), is a highly fatal and rapidly spreading disease of young ducklings and is characterized primarily by hepatitis. Three distinct serotypes of DHAV (DHAV-1, 2 and 3) have been reported (Kim et al., 2007; Tseng et al., 2007; Kim et al., 2008; Liu et al., 2011), and no cross-neutralization has been found among them (Tseng and Tsai, 2007). Currently,

*Corresponding author. E-mail: wap4017@163.com; phone: +8615189910087.

Abbreviations: $\mathrm{AAV}=$ adeno-associated virus; $\mathrm{AAAV}=$ avian adeno-associated virus; DHAV = duck hepatitis A virus; DVH = duck viral hepatitis; ITRs = inverted terminal repeats; rAAAV = recombinant avian adeno-associated virus; UTRs = untranslated regions; $\mathrm{VG}=$ vector genomes
DHAV-1 is the most common, virulent, and widely distributed etiological agent of DVH. DHAV is a member of the new genus Avihepatovirus (the family Picornaviridae). The full genome is about $7.8 \mathrm{~kb}$ (Kim et al., 2006; Ding and Zhang, 2007), and contains a long single open reading frame (ORF) flanked by the $5^{\prime}$ and 3 ' untranslated regions (UTRs). The ORF encodes a polyprotein precursor of 2249 amino acids, which is hydrolyzed into 12 mature polypeptides containing three structural proteins VP0, VP1 and VP3.

DHAV-1 disease has spread worldwide and continues to be a threat to duck farms because of the high mortality of up to $100 \%$. Control of DHAV currently relies on the use of an attenuated vaccine. Though the attenuated vaccine could induce strong immunity and provide enough protection for ducklings (Crighton and Woolcock, 1978), the probability of reversion to virulence still exists. To overcome these limitations, immunization of ducklings against DHAV with 
recombinant genetically engineered vaccines has already been reported; such as the suicidal DNA vaccine (Fu et al., 2012) and the recombinant Pichia pastoris subunit vaccine (Wang et al., 2014).

The adeno-associated virus (AAV), a member of the family Parvoviridae, has been widely used for gene delivery in humans and other species (Wright et al., 2003). The avian adeno-associated virus (AAAV) has proven to be an efficient vector for gene delivery (Perozo et al., 2008; Wang et al., $2008,2009)$. The advantages of this viral vector include no pathogenicity, no interference with maternal antibodies, low immune response against the vector, and the ability to infect a wide variety of cell types (Virag et al., 2009). The possibility of using recombinant AAAV for transgenic expression of immunogenic proteins for poultry vaccination has not been assessed. The aim of this work was to generate recombinant AAAV (rAAAV) expressing the structural protein VP3 of DHAV-1 with baculovirus/insect cells expression system and to assess the ability to stimulate protective immunity in ducklings.

\section{Materials and Methods}

Cells and virus. Spodoptera frugiperda (Sf9; Invitrogen, USA) cells were maintained at $27^{\circ} \mathrm{C}$ in Sf-900 II serum-free medium (Gibco, USA). Primary duck embryo fibroblast cells were prepared from 11-old-day embryos from SPF eggs and grown at $37^{\circ}$ in Dulbecco's Modified Eagle Medium (DMEM; Gibco) supplemented with $10 \%$ fetal bovine serum (FBS; Gibco), 100 units/ml penicillin (Gibco), and $100 \mathrm{mg} / \mathrm{ml}$ streptomycin (Gibco). DHAV-1 strain SH (Shanghai Veterinary Research Institute, Chinese Academy of Agricultural Sciences, China) was propagated in duck embryos as described previously (Song et al., 2012). Virus titer was determined as $10^{4.6}$ lethal median dose $\left(\mathrm{LD}_{50}\right) / 0.2 \mathrm{ml}$. The virus was stored at $-80^{\circ} \mathrm{C}$ until use. The recombinant baculoviruses $\mathrm{rBac}$-Cap and $\mathrm{rBac}-$ Rep, expressing the Cap and Rep proteins of AAAV respectively, were generated as described earlier (Wang et al., 2017) and stored at $4^{\circ} \mathrm{C}$ until use.

Construction of recombinant baculovirus. The VP3 gene of DHAV-1 between nucleotides 1355 and 2065 (GenBank Acc. No. FJ157178) was amplified by PCR from pFB-P13CD (Wang et al., 2018) vector using primers 5 '-gtactcgagaccATGGGAAAGAGA AAACCACGCAAG-3' and 5'- gtagcggccgcTTACTGATTATTG GTTGCCATCTGC-3' (Xho I and Not I sites are underlined; uppercases indicate the VP3 coding sequence), a start codon (ATG) and a stop codon (TGA) were included in the primers, and a Kozak sequence was introduced in front of the start codon to improve the efficiency of VP3 protein translation. The PCR product was cut with $X h o I$ and NotI and cloned downstream of the CMV promoter in a previously developed vector pFBAGFP containing the inverted terminal repeats (ITRs) of AAAV (Wang et al., 2017), and the recombinant vector was designated as pFB-VP3 (Fig. 1). Then the

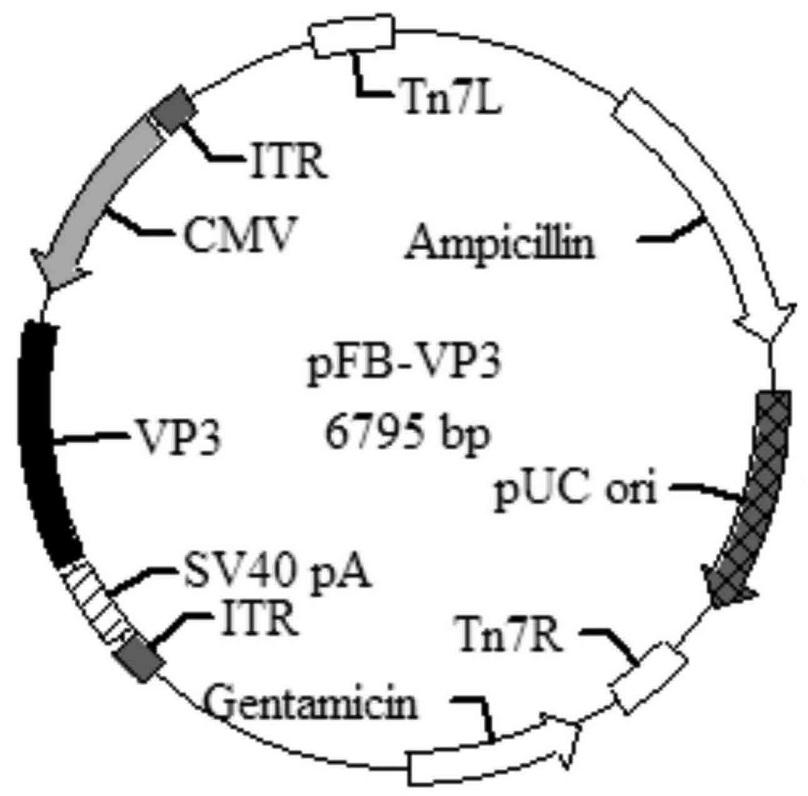

Fig. 1

The construction of baculovirus transfer vector $\mathrm{pFB}-\mathrm{VP} 3$ VP3: structural gene of duck hepatitis A virus type 1 (DHAV-1); ITR: inverted terminal repeats of avian adeno-associated virus (AAAV); CMV: the late cytomegalovirus (CMV) promoter; SV40 pA: simian virus 40 polyadenylation signal; Tn7L: left of mini Tn7 elements; Ampicillin: ampicillin resistance gene; pUC ori: plasmid origin of replication; Tn7R: right of mini Tn7 elements; Gentamicin: gentamicin resistance gene.

recombinant vector $\mathrm{pFB}-\mathrm{VP} 3$ was used to produce recombinant baculoviruses rBac-VP3, according to the manufacterer's instruction of Bac-to-Bac baculovirus expression system (Invitrogen).

Generation of rAAAV-VP3 in insect cells. The rAAAV vector containing VP3 gene, namely rAAAV-VP3, was prepared according to our previously reported method (Wang et al., 2017). Insect cells Sf9 at a density of $2 \times 10^{6}$ cells $/ \mathrm{ml}$ in suspension culture were infected with rBac-Cap, rBac-Rep and rBac-VP3 (MOI 5). About 3 days later, the cells were collected by centrifugation, the rAAAV particles were released by repeated freezing and thawing from $-80^{\circ} \mathrm{C}$ to $37^{\circ} \mathrm{C} 3$ times and purified by PEG precipitation method as described previously (Matsushita et al., 1998). Briefly, the supernatant of the transfected cell lysates was extracted once with $1 / 10$ volume of chloroform and the viral particles in the upper phase were precipitated with $10 \%$ (wt/vol) PEG8000. The sedimented particles were resuspended in PBS and analyzed by electron microscopy. The samples were mounted on a carbon-coated paper grid for 10 min and drained with a filter paper. After being negatively stained with $2 \%$ phosphotungstic acid ( $\mathrm{pH} 7.4$ ) for $1 \mathrm{~min}$, the samples were observed under a transmission electron microscope (TEM; Tecnai 12, FEI, USA). The copy number of vector genomes (VG) was measured by quantitative real-time PCR (qRT-PCR) assay on a 7,300 real-time PCR system (Applied Biosystems, USA). 
Proteinase K-treated rAAAV-VP3 was serially diluted and amplified by PCR, using Maxima SYBR Green/ROX qRT-PCR master mix (Thermo Scientific, USA) with primers specific to the CMV promoter: 5'-ATTTGCGTCAATGGGGCGGAGTT-3' and 5'-CAT CAATGGGCGTGGATAGCGGT-3'. The cycling conditions were as follows: denaturation at $95^{\circ} \mathrm{C}$ for $3 \mathrm{~min}, 40$ cycles of $95^{\circ} \mathrm{C}$ for $15 \mathrm{~s}, 58^{\circ} \mathrm{C}$ for $60 \mathrm{~s}$, followed by dissociation curve determination of the products with melting curve analysis at $95^{\circ} \mathrm{C}$ for $15 \mathrm{~s}, 60^{\circ} \mathrm{C}$ for $15 \mathrm{~s}$, and $95^{\circ} \mathrm{C}$ for $15 \mathrm{~s}$. Linearized pFB-VP3 was employed as a copy number standard.

Detection of VP3 expression by immunofluorescence assay. To prepare the anti-VP3 serum, each mouse was injected intramuscularly three times with $50 \mu \mathrm{g}$ of Escherichia coli expressed DHAV VP3 protein as described previously (Li et al., 2013). Primary duck embryo fibroblast cells were seeded on 24-well plates at a density of $2 \times 10^{5}$ cells/well and infected with rAAAV-VP3. Three days after infection, the cells were fixed for $20 \mathrm{~min}$ at room temperature with $4 \%$ paraformaldehyde and blocked for 60 min with 5\% BSA in PBS. The standard indirect immunofluorescence was performed using mouse anti-VP3 (1:100) serum. After incubation with FITC-labeled goat anti-mouse IgG (1:500, Jackson ImmunoResearch, USA), the cell cultures were observed under microscope for fluorescence.

Detection of VP3 expression by Western blot. Primary duck embryo fibroblast cells were infected with rAAAV-VP3 as described above. The cell pellets were run on 10\% SDS-PAGE and the separated proteins were transferred onto PVDF membranes (Millipore, USA) using Semi-Dry transfer unit (TE70X, Hoefer, USA). The membranes were blocked overnight at $4^{\circ} \mathrm{C}$ with $10 \%$ skimmed milk in PBST and incubated for $2 \mathrm{~h}$ at room temperature with mouse anti-VP3 (1:100) serum. After three washes in PBST, the membranes were incubated for $1 \mathrm{~h}$ with HRP-labeled goat antimouse IgG (1:2,000, Jackson Immuno Research), and the signals were developed with 3, 3'-diaminobenzidine substrate.

Immunization of ducklings and virus challenge. The animal studies were performed in accordance with the animal study protocol of the Institutional Animal Care and Use Committee guidelines set by Jiangsu Agri-animal Husbandry Vocational College (protocol approval No.15-08). One day ducklings were purchased from the National Waterfowl Germplasm Resource Pool of Jiangsu Sci-tech Demonstration Garden of Modern Animal Husbandry, China. The ducklings were randomly divided into three groups of 10 and housed separately. The first group was immunized one time with rAAAV-VP3 intramuscularly (i.m.) at a dose of $10^{9} \mathrm{VG}$ in a volume of $0.5 \mathrm{ml}$. The second group was immunized i.m. with $10^{4.3}$ ELD50/0.5ml commercial attenuated vaccine (A66 vaccine, Nanjing Tianbang Co., China). The third group was injected with PBS as a control. Blood was obtained from five ducklings per group weekly post-immunization. Two weeks after the immunization, the remaining ducklings were challenged i.m. with $0.2 \mathrm{ml}$ of allantoic fluid containing $10^{3} \mathrm{LD}_{50}$ of DHAV-1 strain $\mathrm{SH}$. Three days post challenge, the ducklings were euthanized by intravenous injection of $26 \%$ solution of sodium pentobarbital, the livers were collected and stored at $-80^{\circ} \mathrm{C}$ until use.
Enzyme linked immunosorbent assay. Serum samples were subjected to indirect ELISA test using the E. coli expressed VP3 protein as capture antigen. The VP3 protein was expressed in E. coli BL21 (DE3) using the vector pET-30a, and the recombinant proteins were purified by Ni2+ affinity chromatography His-Bind Resin (Novagen, USA). Briefly, the 96-well microtiter plates were coated overnight with $10 \mu \mathrm{g} / \mathrm{ml}$ recombinant protein VP3, blocked with $1 \%$ BSA for $2 \mathrm{~h}$ at $37^{\circ} \mathrm{C}$, and then incubated for $2 \mathrm{~h}$ at $37^{\circ} \mathrm{C}$ with two-fold serial dilutions of test sera. After washing with PBST, the plates were incubated for $1 \mathrm{~h}$ at $37^{\circ} \mathrm{C}$ with horseradish peroxidase-labeled goat anti-duck IgG (1:2000, KPL, USA). Absorbance was determined at $450 \mathrm{~nm}$ using a Bio-Rad microtiter plate reader.

Quantitative real-time PCR ( $q R T-P C R)$. In order to detect the copy numbers of DHAV-1 in the livers of ducklings challenged with DHAV-1 strain SH, we performed a qRT-PCR assay as described previously (Song et al., 2012). Total RNA was extracted from 50 mg each of the livers using MinBEST Universal RNA extraction kit (TaKaRa, Japan) according to the manufacturer's instructions. Extracted RNA was reverse-transcribed using oligo(dT) 18 primers (TaKaRa) and M-MLV reverse transcriptase (TaKaRa) according to the manufacturer's instructions. Viral RNA expression in the liver of challenged ducklings was determined by qRT-PCR assay on a 7300 real-time PCR system (Applied Biosystems) with primers specific to the 3D gene of DHAV-1: 5'-AAATCAA GGAAGGGAAAACAAGAGGCA-3' and 5'-AACAGCACAAC CAGATATAATGAAAGA-3'. A standard plasmid containing the 3D gene of DHAV-1 was used to establish a standard curve, and a regression curve was constructed plotting the threshold cycle $(\mathrm{Ct})$ values versus the logarithm of the copy number. The qRT-PCR reactions were carried out in 96-well plates in a final volume of $25 \mu \mathrm{l}$, containing $12.5 \mu \mathrm{l}$ of $2 \times$ Maxima SYBR Green/ROX qPCR master mix (Thermo Scientific), $0.3 \mu \mathrm{mol} / \mathrm{l}$ of each primer and $2 \mu \mathrm{l}$ cDNA template or standard template. The cycling conditions were as follows: denaturation at $95^{\circ} \mathrm{C}$ for $3 \mathrm{~min}, 40$ cycles of $95^{\circ} \mathrm{C}$ for $15 \mathrm{~s}, 55^{\circ} \mathrm{C}$ for $60 \mathrm{~s}$, followed by dissociation curve determination of the products with melting curve analysis at $95^{\circ} \mathrm{C}$ for $15 \mathrm{~s}, 60^{\circ} \mathrm{C}$ for $15 \mathrm{~s}$, and $95^{\circ} \mathrm{C}$ for $15 \mathrm{~s}$.

Statistical analysis. Data are expressed as mean \pm SD. The difference among groups was analyzed by Student's $t$-test using SPSS version 13 software. $P$-values of $<0.05$ were considered statistically significant.

\section{Results}

\section{Generation and identification of $r A A A V$-VP3 particles}

The rAAAV-VP3 particles were generated by simultaneous infection of Sf9 cells with rBac-Rep, rBac-Cap and rBacVP3. After purification with PEG8000 precipitation, the viral particles exhibited a typical shape of icosahedron particles with a diameter of approximately $22 \mathrm{~nm}$ under electron microscope (Fig. 2). The qRT-PCR results indicated that the purified rAAAV-VP3 had a titer of $1.05 \times 10^{13} \mathrm{VG} / \mathrm{ml}$. 


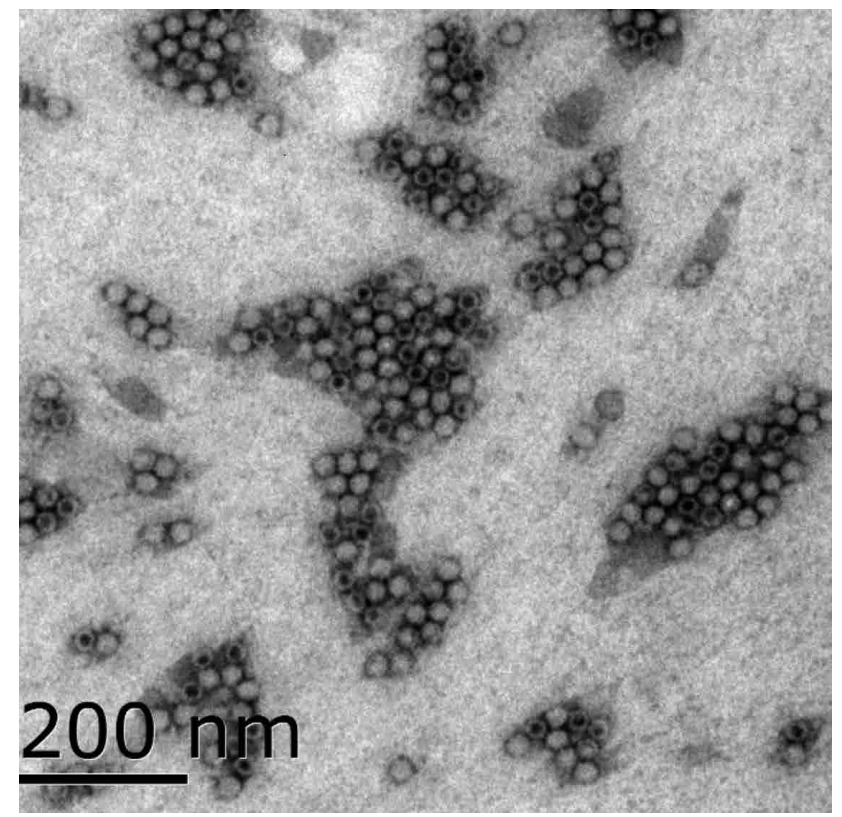

Fig. 2

Electron micrograph of recombinant avian adeno-associated virus (rAAAV-VP3)

Original magnification: $\times 37,000$. The recombinant avian adeno-associated virus (rAAAV-VP3) particles were produced in Sf9 cells, purified with PEG8000 precipitation, and then strained with $2 \%$ phosphotungstic acid. Bar: $200 \mathrm{~nm}$.

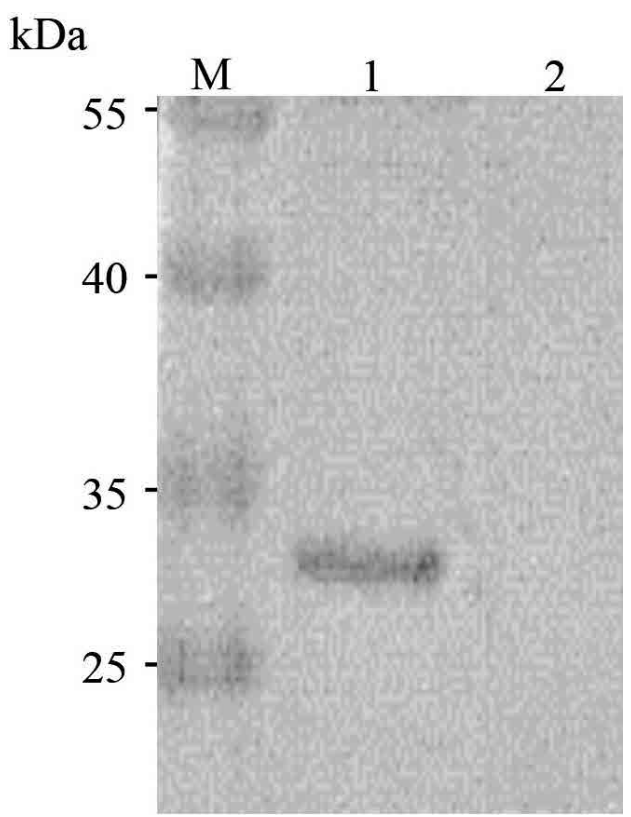

Fig. 3

Western blot analysis of VP3 expression in duck embryo fibroblast cells infected with recombinant avian adeno-associated virus (rAAAV-VP3) Duck embryo fibroblast cells infected with rAAAV-VP3 (1) and normal duck embryo fibroblast cells without viral infection (2) were separated by $10 \%$ SDS-PAGE and detected with mouse anti-VP3 serum and horseradish peroxidase-labeled goat anti-mouse IgG.

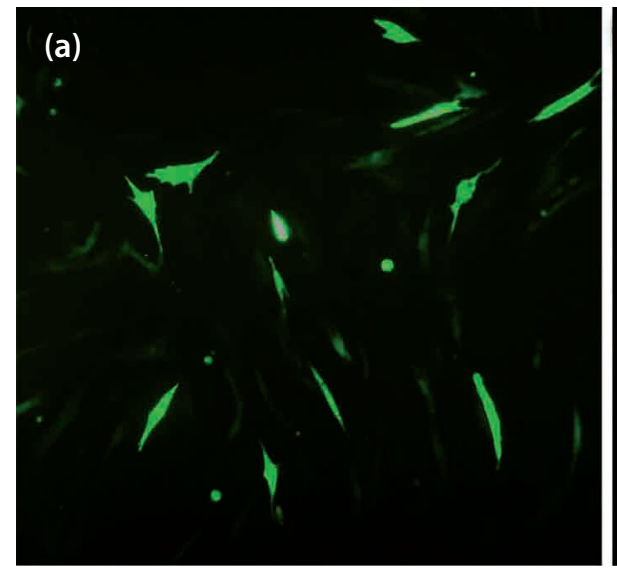

\section{(b)}

Fig. 4

Immunofluorescence assays of VP3 expression in duck embryo fibroblast cells infected with recombinant avian adeno-associated virus (rAAAV-VP3) Three days after rAAAV-VP3 infection, duck embryo fibroblast cells infected with rAAAV-VP3 (a) and normal duck embryo fibroblast cells without viral infection (b) were fixed in $4 \%$ paraformaldehyde and detected with mouse anti-VP3 serum and goat anti-mouse immunoglobulin G labeled with fluorescein isothiocyanate.

In vitro assessment of VP3 expression mediated by $r A A A V$

After infection of primary duck embryo fibroblast cells with rAAAV-VP3, VP3 protein expression was confirmed by immunofluorescence assay using the anti-VP3 antiserum (Fig. 4). Western blot detected the expected $29 \mathrm{kDa}$ protein band in rAAAV-infected cells, but not in mock-infected cells (Fig. 3). 


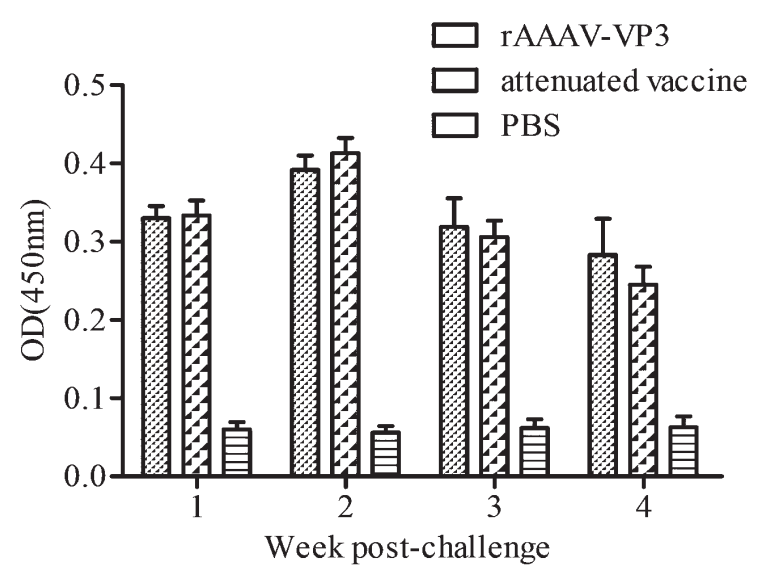

Fig. 5

Titers of IgG antibodies induced in ducklings vaccinated with recombinant avian adeno-associated virus (rAAAV-VP3)

One day ducklings were vaccinated once with rAAAV-VP3 i.m. at a dose of $10^{9} \mathrm{VG}$ or commercial attenuated vaccine. Sera collected at weeks $1,2,3$ and 4 were analyzed for VP3-specific antibody by ELISA using the E. coli expressed VP3 protein as capture antigen. The antisera were diluted 1:64 and used in ELISA. The results were obtained from mean ELISA absorbance values of three sera in each group.

Serological evidence of VP3 protein expression in ducklings

To evaluate the immunogenicity of rAAAV-VP3, one day old ducklings were inoculated i.m. at a dose of $10^{9} \mathrm{VG}$. Blood was collected weekly to test for the presence of anti-VP3 antibody by an indirect ELISA using the E. coli expressed VP3 protein as the coating antigen. The mean antibody level of the vaccinated groups was significantly higher $(0.01<\mathrm{P}<0.05)$ than that of the control group (Fig. 5).

\section{Vaccine/challenge experiment}

To assess the level of protection conferred to ducklings by the $10^{9}$ viral particles of rAAAV-VP3, the livers of the challenged ducklings were collected to detect virus copies by qRT-PCR analysis using the $3 \mathrm{D}$ specific primer. Ducklings of the PBS control group had significantly higher virus copy numbers $(0.01<\mathrm{P}<0.05)$ than the rAAAV-VP3 and the attenuated vaccine inoculated groups, and difference between the $\mathrm{AAAV}-\mathrm{VP} 3$ vaccinated group and the attenuated vaccine inoculated group was not statistically significant (Fig. 6).

\section{Discussion}

The capsid of DHAV is composed of three structural proteins VP0, VP1 and VP3. The VP1 protein has been sufficiently proved to be the major host-protective antigen

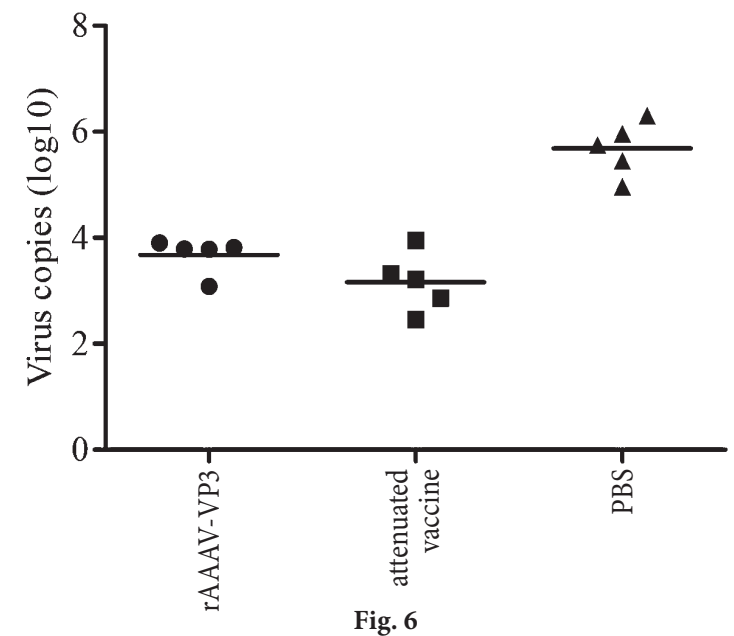

Detection of viral 3D gene expression in livers of challenged ducklings by qRT-PCR

One day ducklings were vaccinated with recombinant avian adeno-associated virus (rAAAV-VP3) i.m. at a dose of $10^{9} \mathrm{VG}$ or commercial attenuated vaccine followed by i.m. injection with $10^{3} \mathrm{LD}_{50}$ of DHAV-1 strain SH two weeks post vaccination. Three days post challenge, the livers were collected for q RT-PCR analysis using 3D specific gene primers. Each symbol represents a duckling, and the solid line indicates the mean value of the group.

containing $\mathrm{T}$ and $\mathrm{B}$ cell epitopes that induce protective neutralizing antibodies (Zhang et al., 2015; Wu et al., 2015; Li et al., 2017). Immunization of ducklings with the recombinant VP1 protein expressed via pichia pastoris (Wang et al., 2014), adeno virus (Yin et al., 2013), and suicidal DNA plasmid (Fu et al., 2012) has been shown to elicit strong protective immunity and provide enough protection against viral challenge (Wang et al., 2014; Fu et al., 2012; Yin et al., 2013). However, there are few studies about structural protein VP3 of DHAV. A cell epitope of 205PSNI208 was identified to be a DHAV-1 type-specific epitope, highly conserved among DHAV-1 (Wu et al., 2017). The 56th amino acid in VP3 of foot-and-mouth disease virus (FMDV), a member of the family Picornaviridae, was reported to play a critical role in virulence and pathogenicity. From all these reports, we hypothesized that the VP3 protein might play an important role in the antigenicity of DHAV-1 and the development of DHAV-1 vaccine. To prove the hypothesis, the VP3 protein was expressed using rAAAV and the immunogenicity was assessed in ducklings.

The AAV is an efficient gene transfer vector for human gene therapy and vaccine research. The rAAAV was first used as the recombinant virus vector to express the VP2 gene of infectious bursal disease virus (IBDV) and could induce protective immunity against IBDV challenge in $80 \%$ of the inoculated chickens (Perozo et al., 2008). There is no other reported study about the $\mathrm{rAAAV}$-based vaccine. The main reason might be that the titer of $\mathrm{AAAV}$ produced with the 
traditional method, co-transfection of HEK293 cells with 3 plasmids expressing the Rep protein, the Cap protein and the foreign genes flanked by the ITRs of AAAV, is too low to meet the clinical demand (Bossis and Chiorini, 2003; Estevez and Villegas, 2004). In our previous study, the rAAAV had been successfully prepared in insect cells using the baculovirus expression system (Wang et al., 2017). The titer of rAAAV was significantly higher than that of rAAAV produced with the traditional method, and the rAAAV has been proven to successfully mediate GFP gene expression in chicken (Wang et al., 2017). In this study, the rAAAV was used as a gene transfer vector to express the VP3 gene of DHAV in duck cells. The VP3 gene of DHAV was subcloned downstream of the promoter CMV of the plasmid pFBAGFP containing the ITRs of AAAV. Assembly of rAAAV-VP3 was detected by electron microscopy, and it was proved that the size of the viral particles corresponds to the normal size of AAAV. The VP3 expression with the corresponding molecular weight of $29 \mathrm{kDa}$ was demonstrated by Western blot analysis of rAAAV-VP3-infected duck embryo fibroblast cells. These results showed that the VP3 gene had been successfully expressed in duck cells and so agreed with our previous observations on the ability of the rAAAV vector to mediate adequate protein expression in vitro (Wang et al., 2008).

Although some papers reported that the VP3 protein contained conserved B cell epitopes (Wu et al., 2017, Shen et al., 2015), there was no evidence to confirm that the VP3 protein could provide enough immune protection in ducklings. To evaluate the immunogenicity of VP3 protein mediated by rAAAV, one day ducklings were vaccinated once with rAAAV at a dose of $10^{9} \mathrm{VG}$ in a volume of $0.5 \mathrm{ml}$. The antibodies were detected by the VP3-specific ELISA test as early as day 7 in all vaccinated ducklings. Statistically significant differences were observed between the vaccinated groups and the nonvaccinated groups. Lethal challenge experiments showed that the level of virus copies in both rAAAV-VP3 group and attenuated vaccine group were significantly lower than that of the PBS group, and the difference of protective immune response in ducklings between the rAAAV-VP3 group and the attenuated vaccine group were not statistically significant. The serological and challenge results observed in the ducklings vaccinated with rAAAV-VP3 in ovo indicated that the VP3 protein expressed in ducklings could induce sufficient immune protection for DHAV challenge. The VP1 protein is the major capsid protein and contains most of the motifs known to interact with cellular receptors and neutralizing antibodies (Oberste et al., 2003), and is the target gene for genetic engineering vaccine design (Wang et al., 2014; Fu et al., 2012). Our results show that the VP3 protein can serve as a candidate for the development of genetic engineering vaccine.

In summary, this experiment demonstrated that the rAAAV-based transgenic expression of the DHAV VP3 protein in ducklings could induce protective immunity against DHAV challenge and presented its potential use in the control of duck viral hepatitis.

Acknowledgments. This work was supported by grants from the Chinese National Science Foundation (31302096), the Agricultural Self-Dependent Innovation Project of Jiangsu Province, China (cx(16)1055), the Natural Science Research Project of Jiangsu Province, China (17KJB230003), the Major Natural Science Research Project of Jiangsu Province, China (18KJA230001). Any of the authors has no conflict of interest to declare for this study.

\section{References}

Bossis I, Chiorini JA (2003): Cloning of an avian adeno-associated virus (AAAV) and generation of recombinant AAAV particles. J. Virol. 77, 6799-6805. https://doi.org/10.1128/ JVI.77.12.6799-6810.2003

Crighton GW, Woolcock PR (1978): Active immunisation of ducklings against duck virus hepatitis. Vet. Rec. 102, 358-361. https://doi.org/10.1136/vr.102.16.358

Ding C, Zhang D (2007): Molecular analysis of duck hepatitis virus type 1 . Virology 361, 9-17. https://doi.org/10.1016/j. virol.2007.01.007

Estevez C, Villegas P (2004): Sequence analysis, viral rescue from infectious clones and generation of recombinant virions of the avian adeno-associated virus. Virus Res. 105, 195-208. https://doi.org/10.1016/j.virusres.2004.05.010

Fu YZ, Chen ZY, Li CF, Liu GQ (2012): Protective immune responses in ducklings induced by a suicidal DNA vaccine of the VP1 gene of duck hepatitis virus type 1 . Vet. Microbiol. 160, 314-318. https://doi.org/10.1016/j. vetmic.2012.06.022

Kim MC, Kwon YK, Joh SJ, Kim SJ, Tolf C (2007): Recent Korean isolates of duck hepatitis virus reveal the presence of a new geno- and serotype when compared to duck hepatitis virus type 1 type strains. Arch. Virol. 152, 2059-2072. https://doi.org/10.1007/s00705-007-1023-0

Kim MC, Kwon YK, Joh SJ, Kwon JH, Lindberg MA (2008): Different diagnosis between type-specific duck hepatitis virus type 1 (DHV-1) and recent Korean DHV-1-like isolates using a multiplex polymerase chain reaction. Avian Pathol. 37, 171-177. https://doi.org/10.1080/03079450801918670

Kim MC, Kwon YK, Joh SJ, Lindberg AM, Kwon JH, Kim JH, Kim SJ (2006): Molecular analysis of duck hepatitis virus type 1 reveals a novel lineage close to the genus Parechovirus in the family Picornaviridae. J. Gen. Virol. 87, 3307-3316. https://doi.org/10.1099/vir.0.81804-0

Li CF, Chen ZY, Meng CC, Liu GQ (2013): High yield expression of duck hepatitis A virus VP1 protein in Escherichia coli, and production and characterization of polyclonal antibody. J. Virol. Methods. 191, 69-75. https://doi.org/10.1016/j. jviromet.2013.04.004

Li XJ, Zhao R, Liu W, Li CX, Zhang TT, Meng FY, Liu M, Zhang Y (2017): Evidence of VP1 of duck hepatitis A type 1 virus as a target of neutralizing antibodies and involving receptor-binding activity. Virus Res. 227, 240-244. https://doi.org/10.1016/j.virusres.2016.10.018 
Liu M, Li XJ, Zhang Z, Liu S, Zhang Y (2011): Goose haemorrhagic hepatitis caused by a new subtype duck hepatitis type 1 virus. Vet. Microbio. 152, 280-283. https://doi. org/10.1016/j.vetmic.2011.05.015

Matsushita T, Elliger S, Eliger C, Podsakoff G, Villarreal L, Kurtzman GJ, Iwaki Y, Colosi P (1998): Adeno-associated virus vectors can be efficiently produced without helper virus. Gene Ther. 5, 938-945. https://doi.org/10.1038/ sj.gt. 3300680

Oberste MS, Maher K, Pallansch MA (2003): Genomic evidence that simian virus 2 and six other simian picornaviruses represent a new genus in Picornaviridae. Virology 314, 283-293. https://doi.org/10.1016/S0042-6822(03)00420-3

Perozo F, Villegas P, Estevez C, Alvarado IR, Purvis LB, Williams S (2008): Protection against infectious Bursal disease virulent challenge conferred by a recombinant avian adeno-associated virus vaccine. Avian Dis. 52, 315-319. https://doi.org/10.1637/8122-100207-ResNote.1

Shen YL, Cheng AC, Wang MS, Chen S, Jia RY, Zhu DK, Liu MF, Sun KF, Yang Q, Chen XY (2015): Development of an indirect ELISA method based on the VP3 protein of duck hepatitis A virus type 1 (DHAV-1) for dual detection of DHAV-1 and DHAV-3 antibodies. J. Virol. Methods 225, 30-34. https://doi.org/10.1016/j.jviromet.2015.08.016

Song CP, Yu SQ, Duan YB, Hu Y, Qiu XS, Tian L, Sun YJ, Wang MS, Cheng AC, Ding C (2012): Effect of age on the pathogenesis of DHV-1 in Pekin ducks and on the innate immune responses of ducks to infection. Arch. Virol. 159, 905-914. https://doi.org/10.1007/s00705-013-1900-7

Tseng CH, Knowles NJ, Tsai HJ (2007): Molecular analysis of duck hepatitis virus type 1 indicates that it should be assigned to a new genus. Virus Res. 123, 190-203. https://doi. org/10.1016/j.virusres.2006.09.007

Tseng CH, Tsai HJ (2007): Molecular characterization of a new serotype of duck hepatitis virus. Virus Res. 126, 19-31. https://doi.org/10.1016/j.virusres.2007.01.012

Virag T, Cecchini S, Kotin RM (2009): Production recombinant adeno-associated virus in foster cells: overcoming production limitations using a baculovirus-insect cell expression strategy. Hum. Gene Ther. 20, 807-817. https://doi. org/10.1089/hum.2009.092

Wang AP, Sun HC, Wang JY, Wang YJ, Yuan WF (2008): Recombinant avian adeno-associated virus-mediated oviductspecific expression of recombinant human tissue kallikrein. Poult. Sci. 87, 777-782. https://doi.org/10.3382/ ps.2007-00372
Wang YJ, Sun HC, Shen PP, Zhang XY, Xia XL (2009): Effective inhibition of infectious bursal disease virus replication by recombinant avian adeno-associated virus-delivered microRNAs. J. Gen. Virol. 90, 1417-1422. https://doi. org/10.1099/vir.0.010520-0

Wang AP, Wang YJ, Wu S, Zuo WY, Guo CM, Hong WM, Zhu SY (2017): Efficient production of an avian adenoassociated virus vector using insect cell/baculovirus expression system. J. Virol. Methods 240, 26-31. https:// doi.org/10.1016/j.jviromet.2016.11.005

Wang AP, Gu LL, Wu S, Zhu SY (2018): Duck hepatitis A virus structural proteins expressed in insect cells self-assemble into virus-like particles with strong immunogenicity in ducklings. Vet. Microbiol. 215, 23-28. https://doi. org/10.1016/j.vetmic.2017.12.020

Wang C, Li XK, Wu TC, Wang Y, Zhang CJ, Cheng XC, Chen PY (2014): Recombinant VP1 protein of duck hepatitis virus 1 expressed in Pichia pastoris and its immunogenicity in ducks. Acta. Virol. 58, 333-339. https://doi.org/10.4149/ av $2014 \quad 04 \quad 333$

Wright JF, Qu G, Tang C, Sommer JM (2003): Recombinant adenoassciated virus: formulation challenges and strategies for a gene therapy vector. Curr. Opin. Drug Discov. Devel. $6,174-178$.

Wu XY, Li XJ, Zhang QS, Wulin SZ, Bai XF, Zhang TT, Wang Y, Liu M, Zhang Y (2015): Identification of a conserved B-cell epitope on duck hepatitis A type 1 virus VP1 protein. PLos One 10, e0118041. https://doi.org/10.1371/journal. pone. 0118041

Wu XY, Zhang TT, Meng FY, Guo DC, Yin XC, Wulin SZ, Li CX, Zhang QS, Liu M, Zhang Y (2017): Mapping a typespecific epitope by monoclonal antibody against VP3 protein of duck hepatitis A type 1 virus. Sci. Rep. 7, 1082. https://doi.org/10.1038/s41598-017-10909-7

Yin XC, Liu HY, Chen YH, Liu M, Zhang Y (2013): Construction of recombinant adenovirus expressing VP1 protein of duck viral hepatitis virus I. Chinese Journal of Preventive Vet. Medicine 35, 526-530 (in Chinese, abstract in English).

Zhang R, Zhou G, Xin Y, Chen J, Lin S, Tian Y, Xie Z, Jiang S (2015): Identification of a conserved neutralizing linear B-cell epitope in the VP1 proteins of duck hepatitis A virus type 1 and 3. Vet. Microbiol. 180, 196-204. https://doi. org/10.1016/j.vetmic.2015.09.008 